\title{
Light disturbance with multifocal contact lens and monovision for presbyopia $^{\text {is }}$
}

\author{
Paulo Fernandes ${ }^{\mathrm{a}, *}$, Ana Amorim-de-Sousa ${ }^{\mathrm{a}}$, António Queirós ${ }^{\mathrm{a}}$, Santiago Escandón-Garcia ${ }^{\mathrm{a}}$, \\ Colm McAlinden ${ }^{\mathrm{b}}$, José Manuel González-Méijome ${ }^{\mathrm{a}}$
}

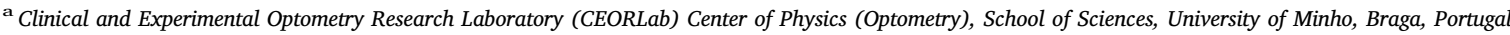

${ }^{\mathrm{b}}$ University Hospitals Bristol, NHS Foundation Trust, Bristol, UK

\section{A R T I C L E I N F O}

\section{Keywords:}

Light disturbance

Multifocal

Monovision

Contact lenses

Presbyopia

\begin{abstract}
A B S T R A C T
Dysphotopsia affects a significant number of patients, particularly after visual correction with multifocal optical designs.

Purpose: Evaluate light distortion (LD) in two modalities of contact lens (CL) wear: multifocal (MF) and monofocal (MV).

Methods: This was a randomized, double-masked, crossover study involving 20 presbyopic patients. Patients were randomized first into either MF or MV for 15 days of use with a 1 week wash-out period between each lens type. The LD was evaluated with the Light Distortion Analyzer (LDA, University of Minho) under monocular and binocular conditions. The light distortion index (LDI, \%), among other parameters were analyzed. Subjective quality of vision was assessed with the Quality of Vision (QoV).

Results: The LD showed an increase in all parameters in both CL modalities being significant for MV in the nondominant eye ( $\mathrm{p}<0.030$, for all LD parameters). For the MF, there was also a significant increase in LDI $(\mathrm{p}=0.016)$ and in BFCrad $(\mathrm{p}=0.022)$ in the non-dominant eye. After 15 days of MF lens wear, there was a significant decrease in all LD parameters $(\mathrm{p}<0.002)$ in the dominant eye. Binocularly, a significant improvement from 1 to 15 days was observed for LDI $(p=0.009)$ and BFCrad $(p=0.0013)$ with MF. The QoV questionnaire showed no significant changes with neither CL. Conclusions. Adaptation to light disturbances induced by MF CL is more effective compared to MV. Practitioners will have greater success if they prepare their patients for the adaptation required as their vision will get better and have less of an issue with light disturbance.
\end{abstract}

\section{Introduction}

A consequence of the progressive ageing of the population is the significant growth in the ng number of contact lens (CL) wearers requiring presbyopic correction. The availability of multifocal CLs, the improved materials/wetting agents and generally better management of dry eyes, together with the improved marketing and familiarity of practitioners with the products, largely contribute to this growth $[1,2]$

Currently, patients have a variety of options for correcting presbyopia with CL, based on different principles: monovision, bifocal or trifocal alternating vision and multifocal simultaneous vision CLs. Within the multifocal lens category, two different types of designs are currently available: concentric spherical or progressive aspheric designs [3,4] Whereas in monovision one eye is corrected for distance and the other eye is enhanced for near vision, [5] multifocal designs of simultaneous vision provide clear vision at various distances, widening the depth of focus of the lens-eye system $[3,6]$.

Monovision modality is independent of pupil size, and vision is lesser compromised in dim lighting or at low contrast [7-9]. On the other hand, the optical principle of multifocal contact lens is based on the formation of multiple images along different foci in each eye, which implies some compromise in visual performance, particularly under low-light conditions $[10,11]$.

Although current multifocal strategies provide satisfactory distance, intermediate and near visual acuity, adverse subjective visual dysphotopic phenomena such as haloes, ghosting, or glare, are often reported by patients fitted with multifocal modalities [12-15].

Positive dysphotopsia is a photic light disturbance (LD) of vision that includes specific phenomena generally described in academic literature as glare, starburst, and haloes. Frequently might also involve hazy vision, monocular diplopia, polyopia, and defocus [16]. Glare refers to a bright and intense light source caused by scattered rays in the

\footnotetext{
A portion of this work has been previously presented at the European Academy of Optics and Optometry Academy 2107 : Barcelona 2017.

* Corresponding author at: Department of Physics (Optometry), School of Science, University of Minho, 4710-057 Gualtar - Braga, Portugal.

E-mail address: pfernandes@fisica.uminho.pt (P. Fernandes).
} 
light path from media opacities and optical discontinuations. It can be divided into discomfort glare and disability glare [17]. The former is a subjective discomfort sensation induced by a bright light without causing significant losses in vision. Disability glare is associated to a significant loss in retinal image contrast due to an inappropriate distribution of light [18]. Halos and starburst, commonly referred as night visual disturbances, degrades the size and shape of the point source of light. Halos are perceived as circular shadows and starburst as a radial or regular scattering of light from a point source [19].

The measurement of these symptoms have been carried out with different methodologies beyond the use of subjective questionnaires and psychometric methods [20-25].

However, some of the commercially available techniques are limited in their ability to discriminate the light disturbance in all directions or do not measure the detailed shape and irregularity features. Previous studies showed an increase of about $15-23 \%$ in light distortion by multifocal intraocular lens (IOL) when compared to monofocal IOL implantation, with trifocal IOL inducing lower values of light distortion than extended depth of focus IOL [26] or bifocal IOLs.15 So far, few studies evaluated the perception of light disturbances in multifocal CL wearers. Besides, the contribution of dominance and the difference in optical design within the same multifocal $\mathrm{CL}$, as well as the binocular summation effect are not completely known [27]. The quantification of such disturbances is relevant to a better understanding of complains and adaptation of simultaneous vision multifocal CL to avoid fitting failure and dropout.

This study aimed to evaluate how different presbyopic corrections with contact lenses affect the LD phenomena. For this purpose, the Light Distortion Analyzer (LDA, CEORLab, University of Minho, Braga, Portugal) $[25,28]$ was used. This device allows measuring light disturbance under more realistic conditions, using hardware with physical LEDs designed to be able to quantify and analyze the size, shape and irregularity of positive dysphotopsia in multiple directions around a central source of glare, under laboratory conditions.

\section{Methods}

\subsection{Sample}

This was a randomized double-masked crossover study involving participants recruited from the staff of the University of Minho and performed at the Clinical and Experimental Optometry Research Lab (CEORLab). Following the tenets of the Declaration of Helsinki, all participants provided informed consent after they received an explanation of the nature, procedures, and consequences of the study. The inclusion criteria were: age between 45 and 65 years; lens opacities under grade II in LOCS III cataract grading scale; maximum spectacle astigmatism of 0.75 diopters (D) in either eye, best-corrected distance visual acuity (VA) of at least $0.00 \log$ MAR in each eye. Patients could not have binocular vision anomaly, no ocular or systemic disease, and no need for medication that might interfere or contraindicate contact lens wear.

Macedo-de-Araújo et al. 29 reported that the induction of $+0.15 \mu \mathrm{m}$ spherical aberration (SA) leads to an increase between 10 and $20 \%$ in light disturbance index (LDI). Considering that the mean induced SA by multifocal CL are in that order of magnitude, the sample size required was 18 subjects, to warrant an $80 \%$ power (type II error risk of $20 \%$ ) and to detect $10 \%$ differences in LDI between follow-up visits, for a statistical significance level of $p=0.05$ (type I error risk of 5\%).

\subsection{Outcome measures}

After confirming subjects' suitability, a crossover study was conducted. Participants were randomized first into either multifocal or monovision for 15 days of wear for each modality with a 1 week washout period between each lens type.
For both modalities, the contact lens used were of silicone hydrogel material (Comfilcon A, Biofinity, Cooper Vision, Fairport, NY) with $48 \%$ of water content, $14.0 \mathrm{~mm}$ diameter and a base curve of $8.60 \mathrm{~mm}$. Subjects were fitted with multifocal (Biofinity ${ }^{\circ}$ Multifocal) contact lenses according to the manufacturer's fitting guidelines for the initial lens selection, and all participants received the same add power in both eyes; the near add power in the non-dominant eye for monovision also matched the add power of multifocal modality.

The Biofinity multifocal combine spherical and aspheric optics to yield a "center-distance" lens for the dominant eye. It comprises a spherical central zone (2.3-mm in diameter) for distance vision, surrounded by a $5.0-\mathrm{mm}$ annular aspheric zone and an $8.5-\mathrm{mm}$ spherical annular zone, both increasing in add power to emphasize distance vision. The "center-near" lens for the non-dominant eye (center-near design) has a $1.7-\mathrm{mm}$ spherical central zone dedicated to near vision followed by a $5.0-\mathrm{mm}$ aspheric annular zone and an $8.5-\mathrm{mm}$ spherical annular zone, both with decreasing add. For monovision, the contact lens used was the single-vision lens (Biofinity) with an aspheric design.

Ocular dominance was identified using the sensory dominance method [30,31], and natural pupil size measured with the NeurOptics ${ }^{\circ}$ VIP $^{\mathrm{TM}}$-200 Pupillometer (Irvine, California, USA) in the same illumination conditions of light distortion measurements.

Once the fitting procedure was completed, subjects were dispensed with the first modality (multifocal or monovision, randomly assigned) and asked to return 14 days later for a follow-up visit to evaluate the fit, vision and comfort, and after a 1-week wash-out period for dispensing the other lens modality. Since the phenomenon of neuronal adaptation to dysphotopsia is unknown, and the time of wear of each lens was short, a 1-week washout seemed as the sufficient time to ensure that adaptive phenomena did not interfere between the different modalities.

All the clinical measurements of visual function were performed $45 \mathrm{~min}$ after finishing the fitting process (day 1 ) and 14 days after (day 15). Visual function analysis was measured using a high-contrast (100\%) and low-contrast (10\%) LogMAR chart (Precision Vision, USA). All VA values reported refer to high (HCDVA) or low (LCDVA) contrast distance VA while HCNVA and LCNVA refer to for high- and low-contrast near VA, respectively. Stereopsis (Stereo Fly SO-001, StereoOptical Co, Inc., Chicago, IL) and contrast sensitivity function (Vision Contrast Test System VCTS 6500, Vistech Consultants, Dayton, OH). Measurements were conducted monocularly and binocularly, under constant photopic $\left(85 \mathrm{~cd} / \mathrm{m}^{2}\right)$ illumination as previously described [7].

\subsection{LDA measurements}

Measurements of light disturbance were performed with an experimental device, Light Disturbance Analyzer (LDA, CEORLab, Portugal) [28]. It consists of central $5 \mathrm{~mm}$ white LED (glare source) surrounded by an array of 240 smaller LED $(1 \mathrm{~mm})$, distributed in twenty-four semi-meridians. These smaller LEDs have a linear separation of $10 \mathrm{~mm}$ to cover an angular field of $10^{\circ}$ at the distance of $2 \mathrm{~m}$. Fig. 1a to c represents the physical arrangement of the device. For technical specifications of the LEDs characteristics and examination procedures consult the previously published work [25,28]. In brief; in a darkened room, the instrument presents the central source of glare at maximum fixed intensity, while the peripheral LEDs are randomly presented at the different semi-meridians. Peripheral LEDs turn-on and turn-off sequentially around the central source of light using different sequences at random times (from 250 to $750 \mathrm{~ms}$ ) and the semi-meridians explored in random order (Fig. 1c). The patient always fixates the central LED and gives feedback when sees the peripheral stimuli by clicking on a remote actuator. Then, the system automatically evaluates the following semi-meridian and examines each semi-meridian three times. If the standard deviation (SD) of these three measurements is above $20 \%$ of the mean value, the device automatically repeats the measurements in those semi-meridians until it reaches values of SD below $20 \%$ of the mean (Fig. 1b). After data collection and storage, a 

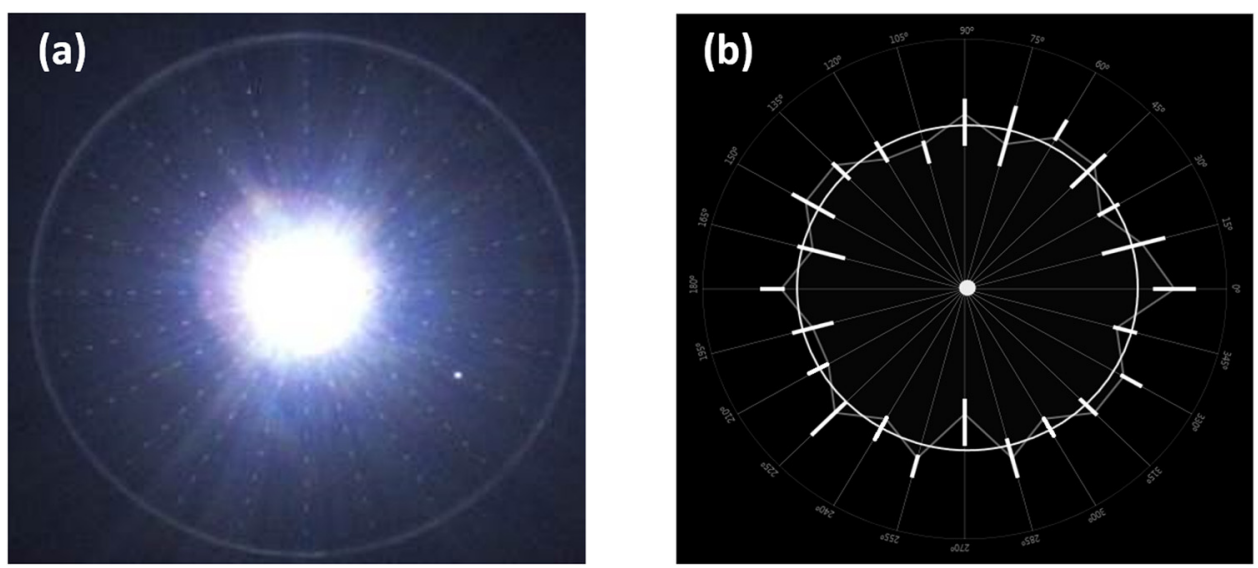

Fig. 1. Illustration of the distribution of main central source of light and peripheral stimuli in accordance with an exemplary embodiment of the present invention. On the above right: the experimental device LDA with the central LED light with one peripheral LED turned ON (a); on the above left an illustration of layout appearance of the size and shape of the light disturbance measured (b) and the size and shape and regular related parameters derived from the Light Distortion Analyzer (c).

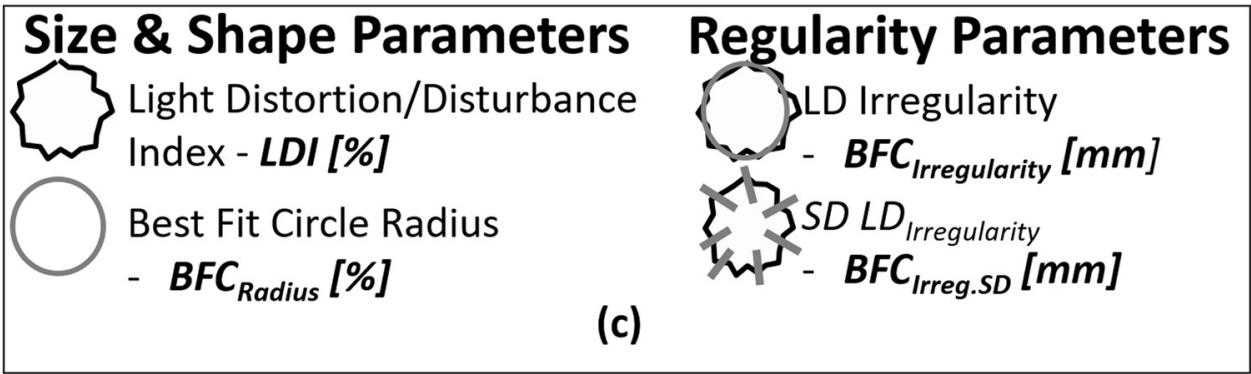

software tool calculates the indices that determine the size, shape, and regularity of the distortion surrounding the central source of light (Fig. 1c). The light disturbance index (LDI) is calculated as the ratio of the area of points missed by the subject and the total area explored, expressed as a percentage (\%). Higher values of distortion represents a lower ability to discriminate surrounding small stimuli hidden by the distortion induced by the central source of light. The Best Fit Circle Radius (BFCRadius) is the circle that best fits the distortion area because of the linear binding of all external points not seen by the subject along each meridian. This parameter linearly correlates with LDI and is expressed in millimeters. The Irregularity of the distortion area derives from the deviation of the actual polygonal shape obtained from the $\mathrm{BFC}_{\text {Radius }}$ fit and is called the $\mathrm{BFC}$ Irregularity ( $\mathrm{BFC}_{\text {Irregularity }}$ ). The standard deviation of $\mathrm{BFC}_{\text {Irregularity }}$ called $\mathrm{BFC}_{\text {Irreg.SD }}$ measures how asymmetric is the departure of the actual distortion limits from the perfectly circular shape of the $\mathrm{BFC}_{\text {Irregularity }}$ Together, $\mathrm{BFC}_{\text {Irregularity }}$ and $\mathrm{BFC}_{\text {Irreg.SD }}$ infer the deviation of the actual distortion from a perfectly rotational symmetric shape. The higher the value of this parameter, the larger the deviation from a circular shape.

\subsection{Quality of Vision (QoV) measurements}

The QoV questionnaire, administered at the final visit for both contact lens modalities, explore the subjective quality of vision [24] afforded by the different contact lens modalities. The QoV questionnaire addresses 10 specific visual symptoms: glare, halos, starbursts, hazy vision, blurred vision, distortion, double or multiple images, fluctuation in vision, focusing difficulties and difficulty in depth perception. The first 7 symptoms have an associated image for easier comprehending. The tool, scored across three subscales, provides a QoV score of frequency, severity, and bothersome nature of symptoms. The scoring is Rasch scaled onto a 0-100 linear scale, with lower scores indicating superior quality of vision [32].

\subsection{Statistical analysis}

Statistical analysis was performed using SPSS for Windows software (version 19, SPSS, Inc.) and the normality of the data checked with the
Kolmogorov-Smirnov test. Friedman test with post-hoc correction was used for the multiple comparisons between the light disturbance at baseline and with each multifocal contact lens (LDI, BFC Radius,

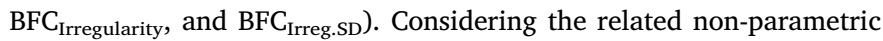
nature of the data, Wilcoxon signed ranks test was applied for pairwise comparison between pairs of multifocal or between baseline measures and each multifocal modality. Spearman Correlation was performed to find the relationship between light disturbance parameters and VA. Differences were considered statistically significant when the $P$ value was less than 0.05 .

\section{Results}

There were twenty-three subjects ( 8 men; 15 women) included in the study. Of the 23 participants enrolled, three ( $1 \mathrm{men} ; 2$ women) were lost to follow-up and excluded, with no data at the 15-day visit with the first randomly assigned modality. Of the 20 subjects who completed the study (mean age, $48.7 \pm 3.3$ years; range, $45-57$ years), $65 \%$ were women and $35 \%$ were myopic (SE $\leq-0.50 \mathrm{D}$ ). Two subjects had previous contact lens experience, but both stopped using them for over a year. Any participant had previously worn presbyopic contact lenses. The mean ( \pm standard deviation) distance spherical equivalent refraction was $-0.91 \pm 2.25$ Diopter (D) (range, -6.88 to +1.63 ) in the dominant eye and $-0.83 \pm 2.35 \mathrm{D}$ (range. $-7.38-2.00$ ) in the non-dominant eye. The mean add power was $+1.5 \pm 0.3 \mathrm{D}$ (five subjects with add of $+1.00 \mathrm{D}, 10$ with add of $+1.50 \mathrm{D}$ and 5 with add $+2.00 \mathrm{D})$. At baseline, the mean pupil size was $5.30 \pm 0.75 \mathrm{~mm}$ and $5.33 \pm 0.79 \mathrm{~mm}$ for the dominant and non-dominant eye, respectively $(\mathrm{p}=0.933)$ and it was $5.32 \pm 0.77 \mathrm{~mm}$ vs $5.28 \pm 0.81 \mathrm{~mm}$ at the $15-$ day visit ( $\mathrm{p}=0.962)$. No significant differences between the pupil size with the two contact lens modalities were found $(p=0.941)$.

\subsection{Visual function}

Binocular VA showed no significant differences neither between the contact lens modalities ( $p>0.05$ in all contrast conditions) nor between follow-up visits ( $p>0.05$ in all comparisons). At the 15-day visit, HCDVA of the dominant eye did not change significantly, from 
$-0.06 \pm 0.08$ LogMar at baseline to $-0.06 \pm 0.08$ LogMar and $-0.05 \pm 0.09$ LogMar in monovision and multifocal, respectively. In the non-dominant eye, it changed from $-0.02 \pm 0.14$ LogMar at baseline to $0.26 \pm 0.16 \operatorname{LogMar}(\mathrm{p}<0.001)$ and $0.16 \pm 0.14$ LogMar $(p<0.001)$ for monovision and multifocal, respectively. LCDVA showed significant changes in the non-dominant eye, which changed from $0.17 \pm 0.15 \mathrm{LogMar}$ at baseline to $0.43 \pm 0.19 \mathrm{LogMar}$ $(\mathrm{p}<0.001)$ and $0.35 \pm 0.13 \operatorname{LogMar}(\mathrm{p}<0.001)$ for monovision and multifocal, respectively. Regarding near visual acuity, HCNVA changed significantly in dominant eye, from $0.10 \pm 0.11$ LogMar at baseline to $0.38 \pm 0.17$ LogMar and $0.20 \pm 0.09$ LogMar in monovision and multifocal, respectively ( $\mathrm{p}<0.001$ all conditions). In the non-dominant eye, it changed from $-0.08 \pm 0.14$ LogMar at baseline to $0.07 \pm 0.13 \operatorname{LogMar}(\mathrm{p}=0.835)$ and $0.06 \pm 0.07$ LogMar $(\mathrm{p}=0.820)$ for monovision and multifocal, respectively. LCDVA showed significant changes in the dominant eye, which changed from $0.28 \pm 0.12 \operatorname{LogMar}$ at baseline to $0.53 \pm 0.18 \operatorname{LogMar}(\mathrm{p}<0.001)$ and $0.37 \pm 0.12 \operatorname{LogMar}(\mathrm{p}<0.001)$ for monovision and multifocal, respectively, while non-dominant eye showed no significant changes $(0.25 \pm 0.08$ LogMar at baseline vs $0.28 \pm 0.15$ LogMar and $0.23 \pm 0.09$ LogMar, $\mathrm{p}=0.232$ ).

From 1 to 15 days of contact lens wear, with multifocal modality, a significant improvement is observed in HCDVA and LCDVA for the nondominant eye ( $p=0.023$ and $p=0.035$, respectively) and in LCNVA in the dominant $(\mathrm{p}=0.026)$ and non-dominant $(\mathrm{p}=0.017)$ eyes.

Mean stereoacuity was $65 \pm 6$ (range: 40-400) sec arc at baseline, $51 \pm 7$ (range: $30-100$ ) sec arc with multifocal and $105 \pm 9$ (range: $40-400)$ sec arc with monovision at the 15 day visit ( $p<0.001$ multifocal vs monovision).

For contrast sensitivity function, all the values remained within the normal range and there were no significant changes after multifocal or monovision compared to baseline, nor between both contact lens modalities ( $\mathrm{p}>0.005$ between all spatial frequencies).

\subsection{Light distortion}

Fig. 2 shows the changes over time of LD size-related parameters: LDI (Fig. 2a) and $\mathrm{BFC}_{\text {Radius }}$ (Fig. 2b), as well as LD regularity-related parameters - $\mathrm{BFC}_{\text {Irregularity }}$ (Fig. 2c) and $\mathrm{BFC}_{\text {Irreg.SD }}$ (Fig. 2d) - for each contact lens modality. In general, when compared to baseline and measured monocularly, there was an increase in all LD parameters with particular significance in the non-dominant eye for both contact lens modalities.

With monovision, dominant eye showed no significant differences in $\operatorname{LD}$ ( $p>0.001$, for all parameters) while non-dominant eye showed a significant increase in LDI $(\mathrm{p}=0.010)$ and $\mathrm{BFC}_{\text {Radius }}$ parameters ( $p=0.014)$. Moreover, at the 15 -day visit, $\mathrm{LDI}$ and $\mathrm{BFC}_{\text {Radius }}$ still significantly higher compared to baseline $(p=0.010$ and $p=0.009$, respectively) and showed no trend to decrease (Fig. 2a and b).

An increase in LD was also observed with Biofinity multifocal, being insignificant in the dominant eye ( $\mathrm{p}>0.05$, for all LD parameters) and significant for $\mathrm{LDI}(\mathrm{p}=0.002), \mathrm{BFC}_{\text {Radius }}(\mathrm{p}=0.004)$ and $\mathrm{BFC}_{\text {Irregularity }}$ $(\mathrm{p}=0.012)$ in the non-dominant eye. In contrast, a significant decrease in $\mathrm{LD}$ was observed with Biofinity multifocal in the Dominant eye ( $\mathrm{p}<0.024$, for all parameters) and for $\mathrm{BFC}_{\text {Radius }}$ in the non-dominant eye $(\mathrm{p}=0.042)$ from 1 to 15 days CL wear, as shown in Table 1 .

Binocular LD parameters showed an initial increase with Biofinity multifocal ( $p<0.040$ for all LD parameters). However, there were a significant improvement in LDI $(\mathrm{p}=0.009)$ and $\mathrm{BFC}_{\text {Radius }}(\mathrm{p}=0.013)$ from 1 to 15 days of multifocal CL wear (Table 1).

With Biofinity multifocal in the dominant eye, a moderate significant positive correlation was found between LDI and LCDVA (Rho $=0.552, \quad \mathrm{p}=0.014)$ and for $\mathrm{BFC}_{\text {Radius }}$ with LCDVA ( $($ Rho $=0.523, p=0.022)$, while a significant negative correlation was found between $\mathrm{BFC}_{\text {Irregularity }}$ and HCNVA (Rho $=-0.561, \mathrm{p}=0.013$ ).

\subsection{Quality of Vision (QoV) questionnaire results}

Fig. 3 shows the results of the QoV questionnaire at 15 days of CL wear. The increases in QoV scores observed for all three subscales (frequency, severity and bothersome) were neither statistically significant compared to baseline correction ( $\mathrm{p}>0.141$ for all the three subscales with both CL modalities), nor significantly different between monovision and Biofinity multifocal in either parameter computed (p $>0.133$ for all the three subscales) (Fig. 3). To better explore the nature of the slight worsening in quality of vision with the contact lens, the scores for glare, halos and starbursts symptoms were analyzed individually (Fig. 4). Although not significant, these symptoms were greater with both CL modalities, with Biofinity MF presenting the higher scores for frequency, severity and bothersome symptoms of glare and starburst ( $\mathrm{p}>0.481$ for all computed scores).

\section{Discussion}

The present study showed an increase in LD phenomena induced by the presbyopic contact lens corrections, despite the good levels of visual acuity, namely under high contrast conditions. This increase in LD phenomena induced by the contact lens is more significant when measured monocularly and has particular incidence in the non-dominant eye. However, there was a significant decrease with the Biofinity multifocal after 15 days of wear, almost reaching baseline values.

Regarding distance and near VA under high- and low- contrast conditions in both modalities, the average binocular HCDVA with both lens types was, two letters worse than the best-corrected spectacle VA, and the binocular VA was always better than the monocular VA under all contrast conditions. Stereoacuity was significantly poorer with monovision rather than with the multifocal contact lens, which was expected because the effects of monocular blur produced by monovision on stereoacuity are known to be greater than the effects of binocular blur (e.g., superimposed retinal images in multifocal lenses). These findings are in agreement with previous studies with identical presbyopic correction [7-9,27,33] and support the existence of a binocular summation effect that attenuates the LD phenomena under binocular conditions, reducing the symptoms reported by subjects. An interesting finding is that such binocular attenuation of LD occurs in a similar amount in monovision and multifocal conditions. Considering the asymmetric vision in the monovision modality, it would expect the summation effect to be lower compared to multifocal conditions. Such disruption of summation effect may be caused by the significantly different levels of spherical aberration induced contralaterally, as reported by Jimenez et al. [34] in anysometropic participants undergoing LASIK surgery. However, with Biofinity multifocal, both eyes received significant different images through the center-distance design and centernear design, which might explain the similar results of the summation effect between monovision and multifocal.

Typically, multifocal CL show a reduction in retinal image quality and contrast VA under mesopic and photopic conditions compared to single-vision contact lenses or spectacles $[8,9]$. The discontinuity between different zones or multiple abrupt changes in the power profile may significantly increase light scatter and result in an increase of light disturbance, particularly under low-light conditions and with the increase in pupil size. In the present study, the two multifocal designs (center-distance vs center-near) differently affected light distortion. The increase in the LD parameters was more noticeable and significant in the non-dominant eye that was fitted with center-near design, in the case of Biofinity multifocal, or with the near vision correction in the case of monovision.

Sivardeen et al. [27] evaluated glare on four different multifocal CL designs (Air Optix Aqua, PureVision 2; Acuvue OASYS and Biofinity MF) using an iPad based halometer (Aston Halometer, Aston University) [22], which quantify the radial glare in eight meridians by recognizing a $0.3 \log M A R$ equivalent letter that is moving eccentrically 

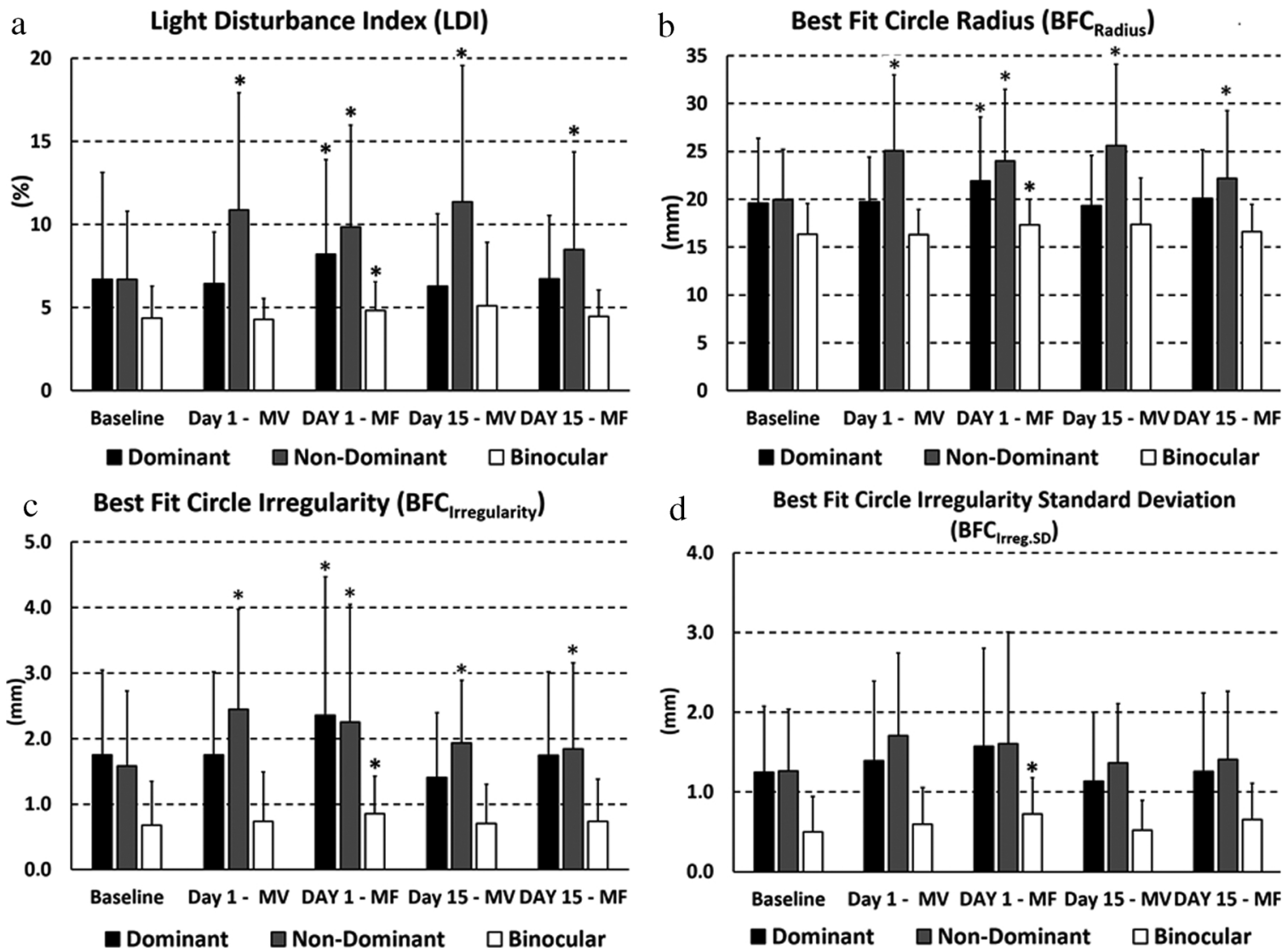

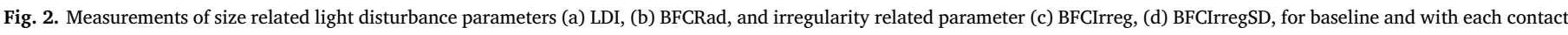
lens modality at the 1st day and 15th day visit. Error bars represent standard deviation. * Wilcoxon-Signed Rank test.

Table 1

Statistically significant changes in parameters of Light Disturbance for Biofinity MF overtime (mean difference \pm SEM)

\begin{tabular}{|c|c|c|c|c|}
\hline & & Eye & Mean change \pm SEM & $P$ value \\
\hline & & & Multifocal & \\
\hline \multirow[t]{2}{*}{ LDI (\%) } & $\begin{array}{l}1 \text { day versus } \\
15 \text { days }\end{array}$ & Bin & $-0.38 \pm 0.20$ & 0.009 \\
\hline & $\begin{array}{l}1 \text { day versus } \\
15 \text { days }\end{array}$ & Dom & $-1.47 \pm 0.65$ & 0.005 \\
\hline \multirow[t]{3}{*}{ BFCRadius (mm) } & $\begin{array}{l}1 \text { day versus } \\
15 \text { days }\end{array}$ & Bin & $-0.71 \pm 0.38$ & 0.013 \\
\hline & $\begin{array}{l}1 \text { day versus } \\
15 \text { days }\end{array}$ & Dom & $-1.86 \pm 0.78$ & 0.012 \\
\hline & $\begin{array}{l}1 \text { day versus } \\
15 \text { days }\end{array}$ & NDom & $-1.83 \pm 0.91$ & 0.042 \\
\hline BFCIrreg (mm) & $\begin{array}{l}1 \text { day versus } \\
15 \text { days }\end{array}$ & Dom & $-0.61 \pm 0.36$ & 0.024 \\
\hline
\end{tabular}

LDI = light distortion index; BFCRadius = Best Fit Circle Radius; BFCIrreg $=$ Best Fit Circle Irregularity; Bin = binocular; Dom $=$ dominant; NDom $=$ non-dominant. $\quad$ * Friedman test; ${ }^{X}$ WilcoxonSigned Rank test.

from a central source. They reported that OASYS and AirOptix design produces the largest halo when measured binocularly, whereas the PureVision 2 created the smallest halo. Similarly to this study, they also found no differences between the Biofinity MF and monovision lenses. There are no differences in LD induced by Biofinity multifocal or monovision in the variations of size- related parameters, measured by the $\mathrm{LDI}$ and $\mathrm{BFC}_{\text {Radius }}$, neither in irregularity-related parameters, measured by the $\mathrm{BFC}_{\text {Irregularity }}$ and $\mathrm{BFC}_{\text {Irreg.SD }}$, despite the slight increase, when compared with the baseline correction.

In the study of Sivardeen et al. [27], the halo was binocularly

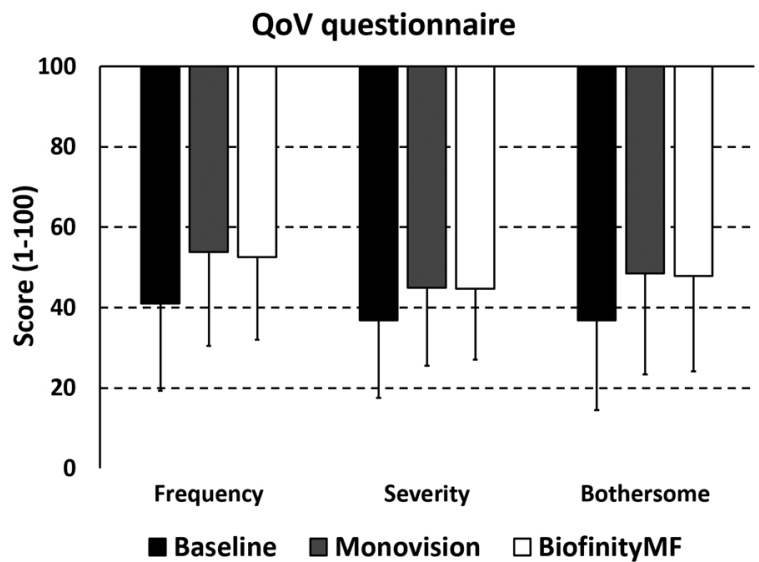

Fig. 3. Rasch-scaled total scores on frequency, severity and bothersome derived from the Quality of Vision (QoV) symptoms with Monovision and Biofinity Multifocal and habitual correction (baseline). The scale extends from 0 to 100, with higher scores indicating worse quality of vision. Error bars represent standard deviation.

measured only, and the contribution of dominance and different design within the same multifocal CL was not evaluated nor the binocular summation effect. In this study, monocular results of LD were always worse than the binocular ones, which may suggest that there is a psychophysical capacity to reduce the LD under binocular conditions and consequently improve the optical quality of LD perception. Binocular vision improves the visual perception of out-of-focus images to a much greater extent than for focused images [6,35]. It seems that with the effect of wearing the multifocal for longer, perceptual processes, such as binocular sum and neural adaptation to aberration changes, enhances the interpretation of multiple images superimposed on the retina 


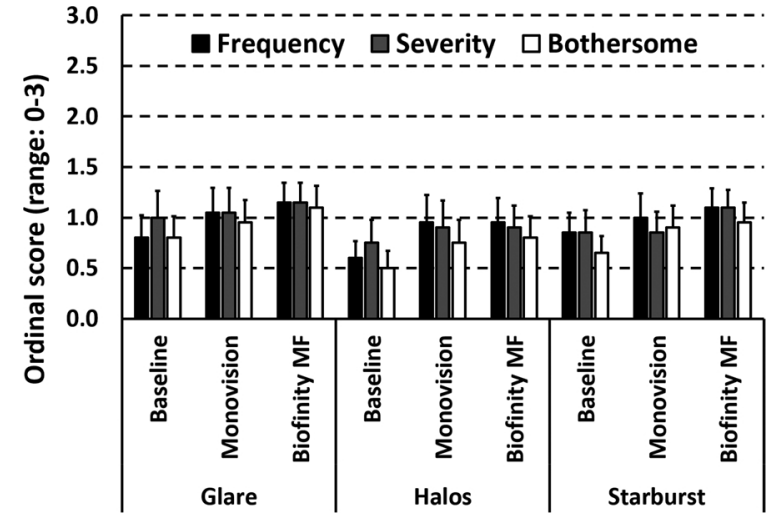

Fig. 4. Frequency, severity and bothersome scores of individual visual symptoms glare (left), halo (middle) and starburst (right) with Monovision and Biofinity Multifocal and habitual correction (baseline). Error bars represent standard errors of the mean.

[7,33]. This may explain the significant decrease in LD observed for Biofinity multifocal, particularly in the dominant eye. Furthermore, the moderate correlations observed between LD parameters and VA suggest that a reduction in the LD parameters may be associated with the improvement in distance and near VA, particularly in low- contrast observed at 15 days of multifocal contact lens wear.

Recent studies showed that wearing multifocal contact lens induces an increase in high-order ocular aberrations (HOA) which is dependent on the multifocal design and differently affect visual performance. $[7,33,36-38]$ Indeed, spherical aberration (SA) is the main HOA induced. While center-near MFCL induces large amounts of negative SA, center-distance MFCL induce an increase in positive SA [31,36]. Moreover, it has been reported that higher amounts of positive SA significantly increase LD, while a lower increase or even decrease can be found with negative SA [39-41]. On one level, this may explain the differences observed in LD between dominant (center-distance design) and non-dominant (center-near design). However, in contrast to the results of Macedo-de-Araujo et al. [29], it was found that the increase in LD was greater with the center-near design (more negative SA) compared to center-distance design. This may be due to the difference for SA induced by multifocal design compared to those experimentally induced in previous studies. Additionally, the subjects of present study were presbyopic, who commonly have smaller pupils and may have some residual accommodation that can compensate some of the degradation induced by HOA and somehow attenuate the LD effect. Nevertheless, this needs further investigation with a larger presbyopic population and with different multifocal contact lens designs.

Some studies reported that the optical properties of different CL materials might affect ocular straylight [41,42]. In the present study, the contact lens material (Comfilcon A) for both presbyopic CL correction were the same to eliminate/minimize the material effect on the $\mathrm{LD}$ and to ensure that the changes were only due to CL design. Increase in LD overtime might also be related to the presence of deposits or to hydrophobic areas at the surface, or degradation of the material bulk of the material. Nevertheless, all participants used hydrogen peroxide and the lenses replaced much earlier than the expected lifetime of 1 month. This might explain the stable behavior or even a decrease observed with multifocal modality, instead of an increase.

Despite the changes observed in LD index, no significant changes were observed with the QoV questionnaire. The QoV questionnaire did not show (any) significant change in QoV score from baseline for either modality of the lens and there were no differences between monovision and multifocal lenses. Recently, Kang et al. [43] assessed the effects of soft multifocal contact lens used for myopia control (Proclear multifocal, CooperVision, with addition power +1.50 and +3.00 ) on subjective quality of vision compared to a single vision Proclear spherical contact lens. They reported a significant increase in QoV scores with
Proclear multifocal with symptoms worsen over the 2-week wearing period, particularly with the multifocal with +3.00 Add. Differences in the higher Add power or the larger pupils of young adults might explain the worse performance when compared to the present study.

In conclusion, multifocal designs and monovision affect differently LD phenomena. The present results show that monovision performs worse than multifocal after 15 days of lens wear. While monocular values experience an improvement from 1 to 15 days with Biofinity multifocal, such did not happen for monovision. Despite the changes observed in LD, subjective symptoms assessed with QoV showed no significant changes. Practitioners will have greater success with multifocal CLs if they prepare their patients for the adaptation required as their vision will get better/have less of an issue with LD. Finally, light distortion measurement does not predict accurately eventual changes in visual acuity and this suggests that it might be used as a different indicator of visual performance and adaptation to multifocal contact lenses.

\section{Acknowledgements}

The authors declare that they do not have any proprietary or financial interest in any of the materials mentioned in this article. JMG-M has property rights on the experimental device used to measure light disturbance. This study has been funded in part by an individual research grant (FCT - SFRH/BPD/92365/2013 to Fernandes, P) and projects PTDC/SAU-BEB/098392/2008 and PTDC/SAU-BEB/098391/ 2008 funded by the Portuguese Foundation for Science and Technology through the European Social Fund and by FEDER through the COMPETE Program and by the Portuguese Foundation for Science and Technology (FCT) in the framework of the Strategic Project UID/FIS/ 04650/2013.

\section{References}

[1] P.B. Morgan, N. Efron, Contact lens correction of presbyopia, Cont Lens Anterior Eye 32 (2009) 191-192.

[2] P.B. Morgan, N. Efron, C.A. Woods, An international survey of contact lens prescribing for presbyopia, Clin Exp Optom 94 (2011) 87-92.

[3] W.N. Charman, Developments in the correction of presbyopia I: spectacle and contact lenses, Ophthalmic Physiol Opt 34 (2014) 8-29.

[4] R. Perez-Prados, D.P. Pinero, R.J. Perez-Cambrodi, D. Madrid-Costa, Soft multifocal simultaneous image contact lenses: a review, Clin Exp Optom 100 (2016) 107-127.

[5] B.J. Evans, Monovision: a review, Ophthalmic Physiol Opt 27 (2007) 417-439.

[6] S. Plainis, G. Ntzilepis, D.A. Atchison, W.N. Charman, Through-focus performance with multifocal contact lenses: effect of binocularity, pupil diameter and inherent ocular aberrations, Ophthalmic Physiol Opt 33 (2013) 42-50.

[7] P.R. Fernandes, H.I. Neves, D.P. Lopes-Ferreira, J.M. Jorge, J.M. González-Meijome, et al., Adaptation to multifocal and monovision contact lens correction, Optom Vis Sci 90 (2013) 228-235.

[8] N. Gupta, S.A. Naroo, J.S. Wolffsohn, Visual comparison of multifocal contact lens to monovision, Optom Vis Sci 86 (2009) 98-105.

[9] K. Richdale, G.L. Mitchell, K. Zadnik, Comparison of multifocal and monovision soft contact lens corrections in patients with low-astigmatic presbyopia, Optom Vis Sci 83 (2006) 266-273.

[10] E. Papadatou, A.J. Del Aguila-Carrasco, J.J. Esteve-Taboada, D. Madrid-Costa, A. Cerviño-Expósito, et al., Objective assessment of the effect of pupil size upon the power distribution of multifocal contact lenses, Int J Ophthalmol 10 (2017) $103-108$.

[11] G. Cardona, S. Lopez, Pupil diameter, working distance and illumination during habitual tasks: implications for simultaneous vision contact lenses for presbyopia, J Optom 9 (2016) 78-84.

[12] B.S. Chu, J.M. Wood, M.J. Collins, Effect of presbyopic vision corrections on perceptions of driving difficulty, Eye Contact Lens 35 (2009) 133-143.

[13] B.S. Chu, J.M. Wood, M.J. Collins, The effect of presbyopic vision corrections on nighttime driving performance, Invest Ophthalmol Vis Sci 51 (2010) 4861-4866.

[14] E.B. Papas, T. Decenzo-Verbeten, D. Fonn, E.B. Papas, T. Decenzo-Verbeten, D. Fonn, B.A. Holden, P.S. Kollbaum, P. Situ, et al., Utility of short-term evaluation of presbyopic contact lens performance, Eye Contact Lens 35 (2009) 144-148.

[15] P. Brito, J. Salgado-Borges, H. Neves, J. Gonzalez-Meijome, M. Monteiro, et al., Light-distortion analysis as a possible indicator of visual quality after refractive lens exchange with diffractive multifocal intraocular lenses, J Cataract Refract Surg 41 (2015) 613-622.

[16] N.S. Jabbur, K. Sakatani, T.P. O'Brien, Survey of complications and recommendations for management in dissatisfied patients seeking a consultation after refractive surgery, J Cataract Refract Surg 30 (2004) 1867-1874. 
[17] D.P. Pinero, D. Ortiz, J.L. Alio, Ocular scattering, Optom Vis Sci 87 (2010) 682-696.

[18] T.J. van den Berg, L. Franssen, B. Kruijt, J.E. Coppens, History of ocular straylight measurement: a review, Z Med Phys 23 (2013) 6-20.

[19] N.I. Fan-Paul, J. Li, J.S. Miller, G.J. Florakis, Night vision disturbances after corneal refractive surgery, Surv Ophthalmol 47 (2002) 533-546.

[20] J.J. Castro, C. Ortiz, A.M. Pozo, R.G. Anera, M. Soler, et al., A visual test based on a freeware software for quantifying and displaying night-vision disturbances: study in subjects after alcohol consumption, Theor Biol Med Model 11 (Suppl. 1) (2014) S1.

[21] C. Villa-Collar, R. Gutiérrez, J.M. González-Méijome, Objective evaluation of night visual distortion, Eur Ophthalmic Rev (2011) 45-47.

[22] P.J. Buckhurst, S.A. Naroo, L.N. Davies, S. Shah, H. Buckhurst, A. Kingsnorth, et al., Tablet App halometer for the assessment of dysphotopsia, J Cataract Refract Surg 41 (2015) 2424-2429.

[23] A. Queiros, C. Villa-Collar, R. Gutiérrez, J. Jorge, J.M. Gonzalez-Meijome, Quality of life of myopic subjects with different methods of visual correction using the NEI RQL-42 questionnaire, Eye Contact Lens 38 (2012) 116-121.

[24] C. McAlinden, K. Pesudovs, J.E. Moore, The development of an instrument to measure quality of vision: the Quality of Vision (QoV) questionnaire, Invest Ophthalmol Vis Sci 51 (2010) 5537-5545.

[25] H. Ferreira-Neves, R. Macedo-de-Araujo, L. Rico-Del-Viejo, A.C. da-Silva, A. Queirós, J.M. González-Méijome, et al., Validation of a method to measure light distortion surrounding a source of glare, J Biomed Opt 20 (2015) 75002-75012.

[26] S. Escandón-García, F. Ribeiro, C. McAlinden, et al., Through-focus vision performance and light disturbances of 3 new intraocular lenses for presbyopia correction, J Ophthalmol 2018 (2018) 6165493.

[27] A. Sivardeen, D. Laughton, J.S. Wolffsohn, Randomized crossover trial of silicone hydrogel presbyopic contact lenses, Optom Vis Sci 93 (2016) 141-149.

[28] J.M. Linhares, H. Neves, D. Lopes-Ferreira, M. Faria-Ribeiro, S.C. Peixoto-de-Matos, J.M. Gonzalez-Meijome, et al., Radiometric characterization of a novel LED array system for visual assessment, J Mod Optic 60 (2013) 1136-1144.

[29] R. Macedo-de-Araujo, H. Ferreira-Neves, L. Rico-Del-Viejo, S.C. de-Matos, J.M. González-Méijome, et al., Light distortion and spherical aberration for the accommodating and nonaccommodating eye, J Biomed Opt 21 (2016) 75003-75008.

[30] D. Lopes-Ferreira, H. Neves, A. Queiros, M. Faria-Ribeiro, S.C. Peixoto-de-Matos, J.M. González-Méijome, et al., Ocular dominance and visual function testing, Biomed Res Int (2013) 238943-238949.
[31] H. Momeni-Moghaddam, C. McAlinden, A. Azimi, M. Sobhani, E. Skiadaresi, et al., Comparing accommodative function between the dominant and non-dominant eye, Graefes Arch Clin Exp Ophthalmol 252 (2014) 509-514.

[32] C. McAlinden, E. Skiadaresi, J. Moore, K. Pesudovs, Subscale assessment of the NEIRQL-42 questionnaire with Rasch analysis, Invest Ophthalmol Vis Sci 52 (2011) 5685-5694.

[33] D. Lopes-Ferreira, P. Fernandes, A. Queiros, J.M. Gonzalez-Meijome, Combined effect of ocular and multifocal contact lens induced aberrations on visual performance: center-distance versus center-near design, Eye Contact Lens (2017) January 04, 2017 - Volume Publish Ahead of Print - Issue - p.

[34] J.R. Jimenez, C. Villa, R.G. Anera, R. Gutiérrez, L.J. del Barco, et al., Binocular visual performance after LASIK, J Refract Surg 22 (2006) 679-688.

[35] S. Plainis, D. Petratou, T. Giannakopoulou, D.A. Atchison, M.K. Tsilimbaris, et al., Binocular summation improves performance to defocus-induced blur, Invest Ophthalmol Vis Sci 52 (2011) 2784-2789.

[36] R. Legras, Y. Benard, H. Rouger, Through-focus visual performance measurements and predictions with multifocal contact lenses, Vision Res 50 (2010) 1185-1193.

[37] P. Gifford, T. Cannon, C. Lee, D. Lee, H.F. Lee, H.A. Swarbrick, et al., Ocular aberrations and visual function with multifocal versus single vision soft contact lenses, Contact Lens Anterior Eye 36 (2013) 66-73.

[38] C. Fedtke, J. Sha, V. Thomas, K. Ehrmann, R.C. Bakaraju, et al., Impact of spherical aberration terms on multifocal contact lens performance, Optom Vis Sci 94 (2017) 197-207.

[39] E. Santolaria-Sanz, A. Cervino, J.M. Gonzalez-Meijome, Corneal aberrations, contrast sensitivity, and light distortion in orthokeratology patients : 1-year results, J Ophthalmol (2016) 8453462-8453468.

[40] C. Villa, R. Gutierrez, J.R. Jimenez, J.M. Gonzalez-Meijome, Night vision disturbances after successful LASIK surgery, Br J Ophthalmol 91 (2007) 1031-1037.

[41] R.A. Applegate, D.H. Jones, Disability glare and hydrogel lens wear-revisited, Optom Vis Sci 66 (1989) 756-759.

[42] I.J. van der Meulen, L.A. Engelbrecht, J.M. van Vliet, R. Lapid-Gortzak, C.P. Nieuwendaal, M.P. Mourits, et al., Straylight measurements in contact lens wear, Cornea 29 (2010) 516-522.

[43] P. Kang, C. McAlinden, C.F. Wildsoet, Effects of multifocal soft contact lenses used to slow myopia progression on quality of vision in young adults, Acta Ophthalmol 95 (2017) 43-53. 